\title{
Evaluación de la conciencia metacognitiva en estudiantes de media desde la clase de ciencias
}

\section{Evaluation of metacognitive awareness in middle school students from sciencclass}

\author{
Alfredo Josué Montes Leguía'. \\ Armando Manuel López Hernández². \\ Luis Carlos Pacheco Lora ${ }^{3}$ \\ Universidad de Córdoba, Colombia
}

\section{RESUMEN}

El presente trabajo investigativo tiene como propósito mostrar el análisis de los resultados obtenidos en la aplicación del Inventario de Habilidades Metacognitivas (MAI) que fue aplicado con el fin de indagar las semejanzas

$1 \quad$ Magister (c) en Didáctica de las Ciencias Naturales. amontesleguia@correo.unicordoba.edu.co, teléfono: 3013378737. https://orcid.org/0000-0003-4337-200X . Facultad de Educación y Ciencias Humanas, Universidad de Córdoba, Colombia.

2 Magister (c) en Didáctica de las Ciencias Naturales. alopezhernandez@correo.unicordobba.edu.co, teléfono: 3045771988. https://orcid.org/0000-0001-7102-696X. https://orcid.org/0000-0003-0676-9120

3 PhD. en Psicología. Magister en Educación con énfasis en Cognición. Especialista en Pedagogía y Didáctica. Psicólogo. Docente de Planta Universidad de Córdoba, Córdoba, Colombia. Departamento de Psicopedagogía. Licenciatura en Educación Infantil. Icpacheco@correo.unicordoba.edu.co. Teléfono: 3156992794. https://orcid.org/00000003-0676-9120 y diferencias de la conciencia metacognitiva de un grupo de 15 estudiantes de media académica de la Institución Educativa La Victoria, con características socioculturales específicas en una clase de ciencias. Esto es parte de la identificación inicial de los procesos que integran el impulso de la conciencia metacognitiva, como plataforma para un planteamiento didáctico. Se denota una metodología cuantitativa descriptiva, en tanto que el MAI es una prueba de autorreporte y sus opciones de respuesta se localizan en una escala Likert. Los resultados mostraron tendencias en algunas categorías de la prueba y disparidades en otras, los cuales fueron objeto de reflexión sobre las habilidades que tienen los estudiantes en cuanto a sus procesos metacognitivos. 
PALABRAS CLAVE: Inventario de Habilidades Metacognitivas (MAI), Conciencia Metacognitiva, Educación en Ciencias, Evaluación.

\section{ABSTRACT}

The purpose of this research work is to show the analysis of the results obtained in the application of the Metacognitive Skills Inventory (MAI) that was applied in order to investigate the similarities and differences of the metacognitive awareness of a group of 15 students of academic average from the La Victoria Educational Institution, with specific sociocultural characteristics in a science class. This is part of the initial identification of the processes that make up the impulse of metacognitive awareness, as a platform for a didactic approach. A descriptive quantitative methodology is denoted, while the MAI is a selfreport test and its response options are located on a Likert scale. The results showed trends in some categories of the test and disparities in others, which were the object of reflection on the abilities that students have in terms of their metacognitive processes.

KEY WORDS: Metacognitive Skills Inventory (MAI), Metacognitive Awareness, Science Education, Evaluation.

\section{INTRODUCCIÓN:}

De manera general, el término "metacognición", se refiere al conocimiento y control que tiene el individuo sobre sus propias actividades de pensamiento y sobre cómo estos conocimientos influyen en la ejecución de las tareas que él realiza.

El dividir la metacognición en categorías ha permitido que los investigadores hayan conceptualizado aún más este constructo. Esa división se conoce comúnmente como, conocimiento y regulación metacognitiva; aunque también se ha teorizado que estas categorías están relacionadas entre sí (Brown, 1987; Flavell, 1987; Campanario \& Moya, 2000).
El conocimiento metacognitivo se puede describir como, lo que saben los estudiantes sobre sus propios procesos cognitivos. Según (Alama Flores, 2015) el conocimiento metacognitivo puede dividirse a su vez en otras unidades, tales como, el conocimiento declarativo donde el estudiante discierne sobre cómo aprende y qué influye en cómo aprende; por su parte, en el conocimiento procedimental sabe sobre las diferentes estrategias 0 procedimientos que funcionan mejor para su aprendizaje y su memoria; y en el conocimiento condicional sabe sobre las condiciones bajo las que puede implementar diferentes estrategias cognitivas. En resumen, diríamos que, un estudiante con conocimiento metacognitivo responde estas preguntas ¿cómo aprendo?, ¿Qué procedimientos o estrategias me resultan más eficaces?, y ¿qué exige de mí, estas condiciones? (Alama Flores, 2015).

Por su parte, la regulación metacognitiva, puede considerarse como las actividades reales en las que participan los estudiantes para facilitar su aprendizaje y memoria (Alama Flores, 2015). La regulación metacognitiva se puede dividir a su vez en tres acciones, tales como, la planificación, el seguimiento o monitoreo y la evaluación. En la planificación, el estudiante precisamente planifica su tarea cognitiva seleccionando las estrategias y recursos cognitivos adecuados; en el seguimiento o monitoreo, el estudiante es consciente de su progreso a través de una tarea cognitiva y su capacidad para determinar su desempeño; y en la evaluación, el estudiante echa un vistazo al resultado y determina si el resultado del aprendizaje coincidió con sus metas de aprendizaje y la efectividad de los procesos de regulación usados (Alama Flores, 2015). Por otra parte, (Brown, 1987; Schraw \& Dennison, 1994; Schraw \& Moshman, 1995) señalaron que la regulación de la cognición incluye otras dos acciones que facilitan el control del aprendizaje, estas son: la organización y la depuración. En la organización, el estudiante 
aplica estrategias y heurísticas que ayudan a gestionar la información; y en la depuración, utiliza estrategias para corregir errores durante el desarrollo de la tarea.

Es lógico pensar que, si los estudiantes han desarrollado un conocimiento $y$ habilidades reguladoras metacognitivas y las usan, favorecerían su aprendizaje. De esta manera se hace importante poder evaluar la metacognición de los estudiantes en el aula de ciencias para determinar en qué nivel de desarrollo se encuentran estos conocimientos y habilidades. Si podemos decir que el conocimiento y las habilidades metacognitivas están presentes en el aula de ciencias, consecuentemente los planteamientos metacognitivos tendrán mayor éxito; tendríamos mayor certeza del aprendizaje de las ciencias y los profesores podrían aplicar diferentes técnicas metacognitivas para evaluar la metacognición de sus estudiantes y desarrollar medios para mejorar la metacognición de los estudiantes cuando sea necesario. (Gula \& Shehzadb, 2012).

Existen pruebas para examinar la metacognición, para este estudio se utilizó El "Metacognitive Awareness Inventory" (MAI) o Inventario de Conciencia Metacognitiva, es un instrumento creado por (G. Schraw \& Dennison, 1994) para identificar habilidades metacognitivas de Conocimiento de la cognición o Regulación de la cognición en los sujetos discutidos anteriormente. El cuestionario ha sido ampliamente utilizado en investigaciones con estudiantes de universidad y en ocasiones adaptado para ser aplicado a docentes, Balcikanli (2011) lo llamó MAIT. También se ha utilizado con niños de primaria, el MAI Junior, por Sperling, Howard, Miller \& Murphy (2002). Cuenta con versiones en diferentes idiomas. En este estudio se utilizó la versión en español, adaptada y validada con estudiantes colombianos para su uso, por Huertas Bustos, Vesga Bravo, Galindo León (2014).

\section{METODOLOGÍA:}

La metodología del presente estudio es cuantitativa descriptiva. El cuestionario se caracteriza por ser de autorreporte tiene 52 afirmaciones que los estudiantes califican como falsas o verdaderas en una escala Likert de cinco puntos, sus opciones de respuesta son los enunciados: 1. Completamente en desacuerdo, 2. En desacuerdo, 3. Ni en desacuerdo ni de acuerdo, 4. De acuerdo y 5. Completamente de acuerdo. Dentro del inventario hay 17 afirmaciones relacionadas con la categoría conocimiento de la cognición y 35 afirmaciones relacionadas con la categoría regulación de la cognición.

El cuestionario está distribuido en ocho subcategorías diferentes: conocimiento declarativo $(5,10,12,16,17,20,32,46)$, conocimiento procedimental $(3,14,27,33)$, conocimiento condicional $(15,18,26,29$, 35), planificación $(4,6,8,22,23,42,45)$, organización $\quad(9,13,30,31,37,39,41,43$, $47,48)$ monitoreo $(1,2,11,21,28,34,49)$, depuración $(25,40,44,51,52)$, y evaluación $(7$, $19,24,36,38,50)$.

Después de ser tomada en cuanta las consideraciones éticas, se determinó aplicar el cuestionario en forma escrita (Ver anexo), por condiciones como ausencia de internet y dispositivos. El espacio en el que se aplicó el cuestionario fue el aula y durante la jornada de clases. Llegado el día y hora de aplicación del cuestionario, se informó a los estudiantes de las características del formato y el objetivo de la aplicación. Se indicó que, las preguntas no hacían parte de ningún requisito de clase, no estaban vinculada a ninguna nota, la participación era voluntaria, no había respuestas correctas o incorrectas, tampoco límite de tiempo para responder, y que sus respuestas debían ser lo más sinceras posible. Todas estas indicaciones, con el fin de que ninguno de estos factores sesgara sus calificaciones en el inventario y 
confundiera los resultados del estudio; todos aceptaron el formato para dar respuestas.

\section{RESULTADOS:}

Los resultados fueron sistematizados a través del uso del software ofimático Excel. Se obtuvieron 780 respuestas de los 15 estudiantes y sus 52 ítems respondidos. Se obtuvo 255 respuestas para la categoría conocimiento metacognitivo y 525 para la categoría regulación metacognitiva.
Para las subcategorías los resultados fueron, para conocimiento declarativo 120 , conocimiento procedimental 60 , conocimiento condicional 75 , planificación 105, organización 150 , monitoreo 105, depuración 75, y evaluación 90. En la siguiente tabla se consolida el número total de respuestas de afirmación o negación de acuerdo con la opción elegida. Se muestran resultados en relación con las categorías de conocimiento y regulación, y del cuestionario

Tabla 1 Consolidado total de respuestas del MAI por categorías.

\begin{tabular}{|c|c|c|c|c|c|c|c|c|c|c|c|c|}
\hline & \multicolumn{2}{|c|}{ 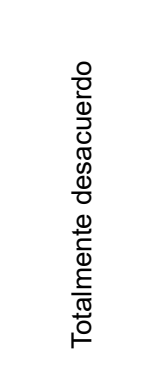 } & \multicolumn{2}{|c|}{ 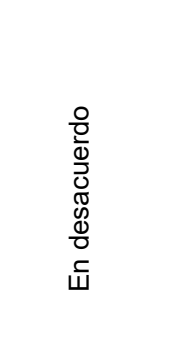 } & \multicolumn{2}{|c|}{ 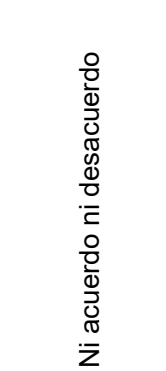 } & \multicolumn{2}{|c|}{ 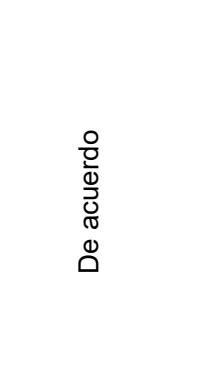 } & \multicolumn{2}{|c|}{ 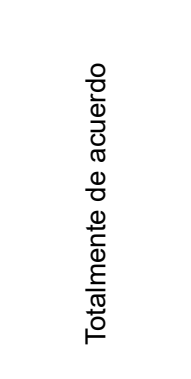 } & \multirow[t]{2}{*}{ बूँ } & \multirow[t]{2}{*}{ 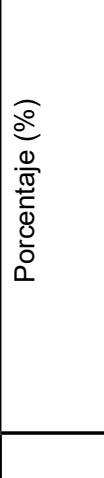 } \\
\hline & $\mathrm{N}^{\circ}$ & $\%$ & $\mathrm{~N}^{0}$ & $\%$ & $\mathrm{~N}^{0}$ & $\%$ & $\mathrm{~N}^{\circ}$ & $\%$ & $\mathrm{~N}^{\circ}$ & $\%$ & & \\
\hline $\begin{array}{l}\text { Conocimiento } \\
\text { metacognitivo }\end{array}$ & 1 & 0,39 & 31 & 12,15 & 23 & 9,01 & 129 & 50,58 & 71 & 27,84 & 255 & 32,69 \\
\hline $\begin{array}{l}\text { Regulación } \\
\text { metacognitiva }\end{array}$ & 10 & 1,90 & 119 & 22,66 & 69 & 13,14 & 245 & 46,66 & 82 & 15,61 & 525 & 67,30 \\
\hline TOTAL, MAI & 11 & 1,41 & 150 & 19,23 & 92 & 11,79 & 374 & 47,94 & 153 & 19.61 & 780 & 100 \\
\hline
\end{tabular}

Si un estudiante respondía con la puntuación más alta (5) a cada afirmación, al sumar las 52 respuestas tendría esa prueba un total de 260 puntos. De acuerdo con lo anterior, la sumatoria total de puntos posibles de la prueba respecto a los 15 estudiantes, para la categoría conocimiento metacognitivo es 1.275 y para la categoría regulación metacognitiva es 2.625; de esta manera la sumatoria de puntos posibles para la prueba es de 3.900 para todo el grupo. Los resultados obtenidos para cada categoría fueron: para la categoría conocimiento metacognitivo es 1.003 (78,66\%) y para la categoría regulación metacognitiva es 1.845 (70,28\%); de esta manera la sumatoria de puntos obtenidos para la prueba fue 2.848 para todo el grupo.

Para la subcategoría conocimiento declarativo se obtuvieron 492 puntos (82\%) de 600 puntos posibles. Para la subcategoría conocimiento procedimental se obtuvieron 215 puntos $(71,66 \%)$ de 300 puntos posibles. Para la subcategoría conocimiento condicional se obtuvieron 296 puntos $(78,93 \%)$ de 375 puntos posibles. Para la subcategoría planificación se obtuvieron 351 puntos $(68,85 \%)$ de 525 puntos posibles. Para la subcategoría organización se obtuvieron 511 puntos $(68,13 \%)$ de 750 puntos posibles. Para la subcategoría monitoreo se obtuvieron 385 puntos (73.33\%) de 525 puntos 
posibles. Para la subcategoría depuración se obtuvieron 293 puntos $(78.13 \%)$ de 375 puntos posibles. Para la subcategoría evaluación se obtuvieron 305 puntos $(67,77 \%)$ de 450 puntos posibles.

Para el conjunto de datos representados en las respuestas de los estudiantes para todo el cuestionario se obtuvo:

- Las medidas de tendencia central (promedio y moda) fue que la suma ponderada entre el número de ítems, es decir, la media final fue de 3,65 , Por otro lado, la opción de respuesta que más se repitió al responder el cuestionario, es decir, la moda fue 4. La suma de las medias obtenidas en cada ítem fue de 189,87. De todas las medias obtenidas en el cuestionario obtuvimos que, en relación con el ítem, 29 estuvieron por encima de la media, y 23 debajo de la media. En relación con los estudiantes 6 estuvieron por encima de la media, y 9 debajo de la media.

- Para las medidas de dispersión (Varianza y desviación estándar) de acuerdo con la suma total de las varianzas y desviaciones obtenidas por cada ítem los resultados fueron 46.80 y 50.05 respectivamente. Indicamos que se calculó, también, la suma de la varianza en relación con los puntajes obtenidos por cada estudiante en la prueba, la varianza total fue 509,84.

Teniendo en cuenta lo anterior, se valoró la consistencia interna del instrumento por medio del estadístico alfa de Cronbach, que es una medida que determina la correlación entre los ítems que componen una escala, tendrá una alta correlación si miden lo mismo; testifica que existe mayor correlación cuando sea mayor el valor alfa de Cronbach (Campo \& Oviedo, 2008; Cervantes, 2005; Cortina, 1993). No se conoce indicaciones específicas para interpretar el alfa de Cronbach en un conjunto de datos, algunos autores afirman que es aceptable cuando el puntaje se encuentra entre 0,6 y 0,8 (Jaramillo
\& Osses, 2012; Jusi, Denise, \& Leonar, 2006; Nunnally, 1987), otros afirman que es aceptable cuando los valores de coeficientes confiables oscilan entre 0,7 y 0,9 (Campo \& Oviedo, 2008; Kaplan \& Saccuzzo, 2005). Para este caso el alfa Cronbach del instrumento fue 0,92 , por lo que se considera adecuado, y es correcto decir que el instrumento refleja consistencia interna. El alfa Cronbach para la categoría conocimiento metacognitivo fue de 0,72 y para la categoría regulación metacognitiva fue de 0,90 .

\section{ANÁLISIS DE RESULTADOS}

Un análisis inicial muestra que las repuestas de los estudiantes en un alto porcentaje fueron positivas a las afirmaciones indicadas en el MAI.

El análisis de profundización se hizo teniendo en cuenta las dos categorías del MAI (Conocimiento de la Cognición y Regulación de la cognición) y sus ocho subcategorías (Conocimiento Declarativo, Conocimiento Procedimental, Conocimiento Condicional, Planificación, Organización, Monitoreo, Depuración, y Evaluación).

Tanto en la Categoría Conocimiento de la Cognición como en la categoría Regulación de la Cognición, las mayores frecuencias y proporciones se ubican con una tendencia favorable en muchos de los ítems de la subescala De acuerdo; por otra parte, en un segundo margen de tendencia favorable se sitúan las frecuencias y proporciones de los ítems de la subescala Totalmente de acuerdo. En suma, los comportamientos de los 15 sujetos valorados tienden prioritariamente hacia estas dos subescalas; en las otras subescalas, los comportamientos son menos frecuentes $y$ dispersos.

Del total de 780 respuesta obtenidas del MAI, 374 correspondieron a la opción de respuesta "De acuerdo" siendo equivalente a un 47,94\%; y se obtuvieron 153 respuestas para la opción de 
respuesta "totalmente de acuerdo" equivalente a un $19.61 \%$.Se obtuvo un $\mathbf{6 7 , 5 5 \%}$ de respuestas afirmativas. Del mismo modo, otro porcentaje menor de respuestas muestra que los estudiantes reportaron respuestas negativas a las afirmaciones indicadas en el MAI. Un 19, $23 \%$ correspondió a la opción de respuesta "En desacuerdo" y un 1,41\% correspondió a la opción de respuesta "totalmente en desacuerdo". Se obtuvo, entonces, un 20,64\% de respuestas negativas. Tenemos entonces que, los estudiantes autoevaluándose reportan un $\mathbf{8 8 , 1 9 \%}$ de Conciencia Metacognitiva. Por otro lado, 92 respuestas fueron para la opción de "ni en acuerdo ni en desacuerdo" Lo que indica un margen del $11,79 \%$ de duda, indiferencia o desconocimiento metacognitivo.

Por un lado, para la categoría conocimiento metacognitivo se obtuvo un total de 255 respuestas, el 50,58\% fueron para la opción de respuesta "De acuerdo" y el 27,84\% fueron para la opción de respuesta "Totalmente de acuerdo", se obtuvo un total de $78,42 \%$ de respuestas afirmativas. Por otro lado, se obtuvo un 12, $15 \%$ para la opción de respuesta "En desacuerdo" y un $0,39 \%$ para la opción de respuesta en "Totalmente en desacuerdo" siendo un total de $12,54 \%$ de respuestas negativas. Indicando entonces que los estudiantes reportan un $90,96 \%$ de conocimiento metacognitivo y un $9,01 \%$ de desconocimiento metacognitivo.

En lo que alude a la subcategoría Conocimiento Declarativo, definido como el conocimiento que tiene un aprendiz sobre su aprendizaje, sus habilidades y el manejo de sus capacidades cognitivas, más del $80 \%$ de los sujetos examinados se ubica en las subescalas $D e$ Acuerdo y Totalmente de Acuerdo en los siguientes ítems: " soy consciente de los puntos fuertes y débiles de mi inteligencia (ítem 5); "cuando me propongo aprender un tema lo consigo" (ítem 20); y, "me doy cuenta si he entendido algo o no" (ítem 32).
El análisis de este conocimiento declarativo, que constituye el conocimiento almacenado en la memoria de tipo conceptual sobre hechos o ideas, sobre el sujeto mismo, sobre las tareas, o sobre las estrategias que utiliza, es evidente que en la mayoría de las respuestas se privilegia una variable de este conocimiento que es la variable del sujeto, es decir, el conocimiento que tenemos de nosotros mismos como aprendices, de nuestras fortalezas y debilidades. En efecto, hay que subrayar que los contenidos: "soy consciente de los puntos fuertes y débiles de mi inteligencia" y "cuando me propongo aprender un tema lo consigo", corresponden a variables personales que pueden incidir positivamente en el logro del aprendizaje. (Domenech Auqué, 2004).

Respecto de la subcategoría Conocimiento Procedimental, señalado como el conocimiento que tiene un sujeto sobre el uso de sus estrategias de aprendizaje; algo más del $80 \%$ de los sujetos examinados se sitúa en las subescalas De Acuerdo y Totalmente de Acuerdo en los siguientes ítems: "Soy consciente de las estrategias que utilizo cuando estudio" (ítem 27) y "Utilizo de forma automática estrategias de aprendizaje útiles" (ítem 33).

El análisis relativo a la subcategoría Conocimiento Procedimental, más relacionado con el conocimiento práctico sobre el uso de estrategias, revela la particular forma cómo estos sujetos advierten con muestras de consciencia sobre las estrategias concretas que usan cuando estudian para lograr que el aprendizaje sea efectivo ("Soy consciente de las estrategias que utilizo cuando estudio"). Al mismo tiempo, perciben la utilización práctica de las estrategias de aprendizaje como una aplicación que puede llegar a convertirse en algo automático ("Utilizo de forma automática estrategias de aprendizaje útiles). (Calero, 2017)

En cuanto a la subcategoría Conocimiento Condicional, distinguido como el conocimiento 
que sostiene un aprendiz de cuándo y por qué utilizar las estrategias de aprendizaje. En los sujetos examinados algo más del $80 \%$ se ubica en las subescalas De Acuerdo y Totalmente de Acuerdo en los subsiguientes ítems: "Aprendo mejor cuando ya conozco algo sobre el tema" (ítem15); "Puedo motivarme para aprender cuando lo necesito (ítem 26); "Uso los puntos fuertes de mi inteligencia para compensar mis debilidades" (ítem 29).

El análisis relacionado con este Conocimiento Condicional, plantea las condiciones bajo las cuales los aprendices desarrollan las capacidades para elegir o ejecutar definidas acciones estratégicas en el aprendizaje; plantea en esta ocasión el carácter determinante de la motivación, principalmente la intrínseca, como motor impulsador de la conducta de aprender ("Puedo motivarme para aprender cuando lo necesito"). El hecho de ser consciente de la importancia de la motivación en el aprendizaje, es una variable que apuntala los conocimientos declarativo y procedimental de los estudiantes. Así mismo, el ser consciente de las fortalezas y debilidades en el uso de las capacidades intelectuales ("Uso los puntos fuertes de mi inteligencia para compensar mis debilidades"), remite a la naturaleza de intencionalidad y control que sugiere el conocimiento condicional (Pressley \& Harris, 2006)many problems that human beings attempt, with some strategies more likely to result in success than others. Understanding effective performance requires understanding the psychology of strategies; promoting human effectiveness at a task requires understanding of the strategies that can accomplish the task and how to develop such strategies among learners. Strategies development has deservedly received much study by cognitive psychologists, with educational psychologists doing much work to detail how affective, behavioral, and cognitive strategies develop, and can be developed, to increase student performance with respect to important academic tasks. In this chapter, we begin with a definition of a I"strategyl" and a brief discussion of constructs related to research in this area, including procedural and declarative knowledge, long- and short-term memory, metacognition, and good information processing. We then turn to important findings from the earliest research on human strategies use, as these are both critical to understanding current research and to the development of further research. What we have learned about strategies use and strategies instruction in academic areas among students in the elementary through secondary grades then becomes the focus of this chapter.,",author”:[\{“dropping-particle":"”,",famil y":"Pressley","given":"Michael","non-droppingparticle":"”,"parse-names":false,"suffix":"”\},\{“dropping-particle":,","family":"Harris","given":"Ka ren R","non-dropping-particle":"',",parse-name s":false,"suffix":"'\}],"container-title":"Handbook of educational psychology","editor":[\{“droppingparticle":"”,"family":"Alexander","given":",",n on-dropping-particle":",",parse-names":false ,"suffix":"”\},\{“dropping-particle":"”,"family":"P. H. $\quad$ Winne","given":,",",non-droppingparticle":",,"parse-names":false,"suffix":"”\}], "id":"ITEM-1","issued":\{“date-parts":[[“2006" ]]\},"page":"265-286","publisher":"Lawrence Erlbaum Associates Publishers.","publisherplace":"Mahwah, NJ","title":"Cognitive Strategies Instruction: From Basic Research to Classroom Instruction.","type":"chapter"\},"uris":[“http://www. mendeley.com/documents/?uuid=9d5793188fe6-36b6-8bfc-1e75ee217d9f"]\}],"mendeley":; “f ormattedCitation":"(Pressley \& Harris, 2006.

Por otro lado, para la categoría regulación metacognitiva se obtuvo un total de 525 respuestas, el $46,66 \%$ fueron para la opción de respuesta "De acuerdo". Y el 15,61\% fueron para la opción de respuesta "Totalmente de acuerdo", teniendo un total de $62,27 \%$ de respuestas afirmativas. Por otro lado, se obtuvo un $22,66 \%$ de respuestas para la opción de respuesta "En desacuerdo" y un $1,90 \%$ de respuesta para la 
opción de respuesta "Totalmente en desacuerdo" teniendo un total de $24,56 \%$ de respuestas negativas. Los estudiantes reportan un 86,83 $\%$ de regulación metacognitiva y un $13,14 \%$ de falta de regulación metacognitiva.

Se evidencia entonces un puntaje más bajo en la categoría regulación metacognitiva qué en la categoría conocimiento metacognitivo, la tabla 10 muestra que, del total de puntos posibles obtenidos para cada categoría, en la categoría regulación metacognitiva obtuvo un puntaje más bajo. Esto sugiere entonces un análisis detallado de esta categoría.

Encontramos que, las subcategorías que reportan puntajes más bajos son las de planificación $(68,85 \%)$, organización $(68,13 \%)$ y evaluación $(67,77 \%)$, reportando puntajes entre 67 y $69 \%$.

Requiriendo actividades orientadas a que el estudiante juzgue prospectivamente (planificar) su tarea cognitiva y seleccione las estrategias y recursos cognitivos adecuados para su próxima tarea cognitiva; Además, juzgue retrospectivamente (evaluar) echando un vistazo al resultado y determinando si el resultado del aprendizaje coincidió con sus metas de aprendizaje iniciales y la efectividad de los procesos de regulación usados. Todo esto mediado por el uso y aplicación de heurísticas que ayuden a gestionar la información. (Klimenko \& Alvares, 2009).

Con referencia a la subcategoría Planificación, definido en función de la manera en que el sujeto determina los tiempos, recursos y metas de estudio, se observa cómo las mayores frecuencias y proporciones en los sujetos examinados, se desplaza del margen de respuesta de las subescalas De Acuerdo y Totalmente de Acuerdo a las subescalas En desacuerdo. Tal como se pudo observar en los ítems $4,6,8,22$, contrario a lo que ocurre en el presente ítem: "Pienso en distintas maneras de resolver un problema y escojo la mejor" (ítem 23); y un poco menos del $80 \%$ afirma "Leo cuidadosamente los enunciados antes de empezar una tarea" (ítem 42).

El análisis de la subcategoría Planificación conduce a la inferencia de que la regulación metacognitiva en esta variable, es favorable a la idea de que los sujetos tengan conocimiento sobre los diferentes procedimientos que pueden utilizar en el proceso de solución de problemas y en la toma de decisiones oportuna para reconocer cuáles son los procedimientos apropiados para cumplir eficazmente con una tareas de aprendizaje ("Pienso en distintas maneras de resolver un problema y escojo la mejor").

En relación con la subcategoría Organización, determinada como la manera en que el aprendiz logra organizar sus actividades en torno a la meta de aprendizaje, con algo más del $80 \%$ en las subescalas De Acuerdo y Totalmente de Acuerdo, los sujetos valorados destacan las siguientes respuestas: "Me pregunto si lo que estoy leyendo está relacionado con lo que ya sé" (ítem 43); por otra parte, con el $80 \%$ responden "Me invento mis propios ejemplos para poder entender mejor la información" (ítem 31).

En lo que se refiere al análisis de la subcategoría Organización, como parte de la regulación de la cognición, las acciones de organización de las actividades en torno al aprendizaje que ejercen los sujetos en este caso, se relacionan con el planteamiento de auto preguntas, lo que constituye una dimensión metacognitiva de autorregulación que pretende que el lector esté al corriente de sí está comprendiendo, también en función de sus aprendizajes previos ("Me pregunto si lo que estoy leyendo está relacionado con lo que ya sé") (López-Chao, Mato-Vázquez, \& Espiñeira, 2017)

Respecto de la subcategoría Monitoreo, definida como el modo en que el aprendiz supervisa su 
proceso de aprendizaje durante el desarrollo de la tarea o con relación al cumplimento de la meta trazada, algo más del $80 \%$ de los sujetos indagados se sitúa en las subescalas De Acuerdo y Totalmente de Acuerdo en los siguientes ítems: "Me pregunto constantemente si estoy alcanzando mis metas" (ítem1); "Cuando aprendo algo nuevo me pregunto si lo entiendo bien o no" (ítem 49).

En el análisis de la subcategoría Monitoreo, la condición de regulación de la cognición recae sobre la forma cómo estos sujetos logran supervisar la ejecución del proceso de aprendizaje durante el desarrollo de tareas. Tal supervisión se manifiesta nuevamente en el planteamiento de autopreguntas, que también se manifestó en la subcategoría anterior; pero en esta subcategoría, las autopreguntas están destinadas a revisar el cumplimiento de las metas de aprendizaje ("Me pregunto constantemente si estoy alcanzando mis metas") y a regular o autoevaluar la comprensión que se ha obtenido cuando se afrontan nuevos aprendizajes ("Cuando aprendo algo nuevo me pregunto si lo entiendo bien o no"). (Coca Fonseca, 2020)

Asumiendo la subcategoría Depuración, precisada como la habilidad que tiene el aprendiz para identificar sus debilidades y realizar los ajustes para mejorar su desempeño y llegar a su meta de aprendizaje, aquellos sujetos examinados que alcanzan algo más del $80 \%$ en las subescalas De Acuerdo y Totalmente de Acuerdo, sobresalen en los siguientes ítems: "Pido ayuda cuando no entiendo algo" (ítem 25); (ítem 52).

Los elementos de análisis de la subcategoría Depuración, sitúan las deducciones sobre la regulación de la cognición en debilidades que los sujetos identifican, tales como, la solicitud de ayuda o apoyo ("Pido ayuda cuando no entiendo algo") y el ajuste de estrategias para mejorar los desempeños ante la presencia de confusiones o vacilaciones en la lectura de contenidos textuales ("Me detengo y releo cuando estoy confundido"). (Young \& Fry, 2008)

Relacionado con la subcategoría Evaluación, que se define como un proceso de autoevaluación donde el aprendiz examina la efectividad de las estrategias implementadas e identifica si llegó a su meta de aprendizaje, se evidencia que los sujetos examinados no alcanzan a superar el $80 \%$ entre las subescalas De Acuerdo y Totalmente de Acuerdo en los ítems de referencia.

Finalmente, en este análisis de la subcategoría Evaluación de la categoría de Regulación de la Cognición, el grupo de sujetos examinados presentó condiciones menos apreciables para evaluarlasestrategias deaprendizajeempleadas. Estas condiciones menos destacadas en la subcategoría Evaluación, pueden afectar los juicios que hace el individuo sobre sus procesos de pensamiento, capacidades y limitaciones y cómo estas se pueden utilizar en una situación o en un contexto específico. (Iriarte Pupo, 2019)

\section{CONCLUSIONES}

La identificación inicial de los procesos que integran la conciencia metacognitiva, como plataforma para un planteamiento didáctico, permite valorar ciertos elementos favorables en las categorías de Conocimiento de la Cognición y Regulación de la Cognición que fueron descubiertos con la aplicación del MAI. Los sujetos muestran estrategias metacognitivas en algunas variables personales del Conocimiento Declarativo; otras formas de automatización y consciencia del uso de estrategias a nivel procedimental y un nivel de consciencia del papel determinante de la motivación y de las fortalezas y debilidades en el uso de las capacidades intelectuales.

Por otra parte, respecto a los resultados en la categoría de Regulación de la Cognición, existen elementos favorables en las dimensiones de 
Organización y Monitoreo donde destacan las estrategias sobre autopreguntas y de supervisión de la propia comprensión y entendimiento de las tareas de aprendizaje. No obstante, los procesos de Planificación, Depuración y Evaluación, requieren mayor entrenamiento. Es decir, es necesario ahondar más en el desarrollo de estrategias pedagógicas y didácticas que fortalezcan la cognición y el aprendizaje autorregulado, en la perspectiva de que los sujetos logren mejores conocimientos sobre los diferentes procedimientos que pueden utilizar en el proceso de solución de problemas y en la toma de decisiones oportuna; así mismo, identifiquen sus debilidades y realicen ajustes efectivos para mejorar su desempeño académico; y por último, puedan valorar o evaluar los resultados de las estrategias practicadas en términos de eficacia de su aprendizaje.

\section{REFERENCIAS BIBLIOGRÁFICAS}

Alama Flores, C. M. (2015). Hacia una didáctica de la metacognición Towards a didactics of the metacognitive Introducción $i$ Cómo operan las facetas o modalidades metacognitivas? Horizonte de La Ciencia, 5(8), 77-86.

Balcikanli, C. (2011). Metacognitive Awareness Inventory for Teachers (MAIT). Electronic Journal of Research in Educational Psychology, 9(3), 1309-1332.

Brown, A. (1987). Metacognition, executive control, self-regulation and other mysterious mechanisms. In R. H. Kluwe W. (Ed.), Metacognition, motivation and understandin (Lawrence E, pp. 65-116). Hillsdale, NJ.

Calero, A. (2017). Comprensión lectora. Estrategias que desarrollan lectores autorregulados. Madrid: comprensionlectora.org.

Campanario, J. M., \& Moya, A. (2000). ¿Cómo enseñar ciencias? Principales tendencias y propuestas. Enseñanza de Las Ciencias: Revista de Investigación y Experiencias Didácticas, 17(2), 179-192.

Campo, A. \& Oviedo, H. (2008). 'Propiedades psicométricas de una escala: la consistencia interna. Revista de salud pública 10, 5, 831-839.

Cervantes, V. (2005). 'Interpretaciones del coeficiente alpha de Cronbach'. Avances en medición 3, 9-28.

Coca Fonseca, M. A. (2020). Habilidades metacognitivas para la resolución de problemas en el contexto de la enseñanzaaprendizaje de las ciencias.

Cortina, J. (1993). 'What is Coefficient alpha?' Journal of Applied Psychology 78, 1, 98104.

Domenech Auqué, M. (2004). El papel de la inteligencia y de la metacognición en la resolución de problemas. Departament de Psicología, Universitat Rovira i Virgili (UVR). Fichero de tesis.

Flavell, J. (1987). Speculations About the Nature and Development of Metacognition. In F. E. Weinert\&R. H. Kluwe(Eds.), Metacognition, Motivation, and Understanding (pp. 2129). Hillsdale, NJ: Lawrence Erlbaum.

Gula, F., \& Shehzadb, S. (2012). Relationship between metacognition, goal orientation and academic achievement. Procedia Social and Behavioral Sciences, 47, 18641868.

Huertas Bustos, A. P., Vesga Bravo, G. J., \& Galindo León, M. (2014a). Validación del instrumento 'inventario de habilidades metacognitivas (Mai)' con estudiantes colombianos. Praxis \& Saber, 5(10), 55. https://doi.org/10.19053/22160159.3022 
Iriarte Pupo, A. J. (2019). Estrategias metacognitivas en la resolución de problemas matemáticos en estudiantes de $5^{\circ}$ de básica primaria. In G. Poveda, Roa \& Alvarado, Pinilla \& Vesga (Ed.), Análisis del discurso matemático escolar. Sincelejo.

Jaramillo, S. \& Osses, S. (2012). 'Validación de un instrumento sobre metacognición para estudiantes de segundo ciclo de educación general básica'. Estudios Pedagógicos XXXVIII, 2, 117-131.

Jusi, H.; Denise, D. \& Leonar, R. (2006). 'Perceived Third-Person Effects and Consumer Attitudes on Prevetting and Banning DTC Advertis'. The Journal of Consumer Affairs 40, 1, 90-116.

Kaplan, R. \& Saccuzzo, D. (2005). Psychological testing: Principles, applications and issues. Wadsworth: Thomson.

Klimenko, O., \& Alvares, J. (2009). Aprender cómo aprendo: la enseñanza de estrategias metacognitivas. Educación y Educadores, 12(2). Recuperado de https:// educacionyeducadores.unisabana.edu.co/ index.php/eye/article/view/1483/1652

López-Chao, V. A., Mato-Vázquez, D., \& Espiñeira, E. (2017). Impacto del uso de estrategias metacognitivas en la enseñanza de las matemáticas. Perfiles educativos, 91-111.

Nunnally, J. (1987). Teoría psicométrica. México: Trillas. Oviedo, H. \& Campo, A. (2005). 'Aproximación al uso del coeficiente de alfa de Cronbach'. Revista Colombiana de Psiquiatría XXXIV, 4, 2005.

Pressley, M., \& Harris, K. R. (2006). Cognitive Strategies Instruction: From Basic Research to Classroom Instruction. In Alexander \& P. H. Winne (Eds.), Handbook of educational psychology (pp. 265-286).
Retrieved from https://psycnet.apa.org/ record/2006-07986-012

Schraw, G., \& Dennison, R. (1994). Assessing metacognitive awareness. Contemporary Educational Psychology, 19, 460-475.

Schraw, Gregory, \& Moshman, D. (1995). Metacognitive Theories. Educational Psychology Review, 7, 351-371.

Sperling, R.; Howard, B.; Miller L. \& Murphy, C. (2002). 'Measures of Children's Knowledge and Regulation of Cognition'. Contemporary Educational Psychology 27, $51-79$

Young, A., \& Fry, J. (2008). Metacognitive awareness and academic achievement in college students. Journal of the Scholarship of Teaching and Learning, 8, 1-10. 


\title{
Anexo 1 Cuestionario de MAI de autorreporte
}

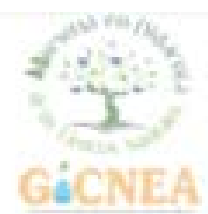

\author{
Estudio de los Juicios Metacognitivos en el \\ Aprendizaje de las Ciencias Naturales
}

Objetive: Analiar la conciencia meracognitiva de los eurudianses de grads dicime de acuerdo a las categorias; cobocimients declurativo, coescimiente procedimestal, ssestimiento conditiseal, planificación, organizacib́s, monitsreo, depuracibe y evaloación, de acuendo al 'Inventario de Hatilidades Metacegaitivas (MAA)'

\section{Inventario de Habilidades Metacognitivas}

Valialadoresu Mustas Bestos, A. Vesga Bewre, G. Galindo Lebin, M. (A0t6)

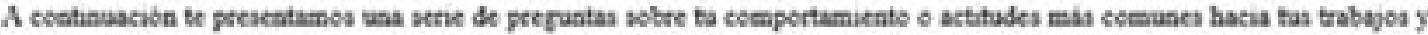

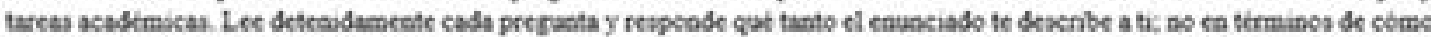
piensas que deberia ser, o de lo que otros piensa de ti. No hay respuestai correctas o incorrectas. Tua sespuestas sería absolutameate confidenciales y únicaeneate serain enpleadas para propónitos investigativos. Por favor contesta todos los

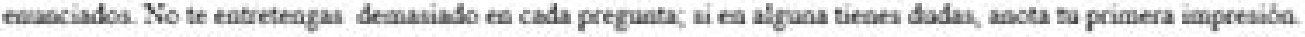

\begin{tabular}{|c|c|c|c|c|}
\hline 1 & 2 & 3 & 4 & 5 \\
\hline 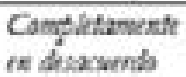 & En desarerds & $\begin{array}{l}\text { Nit en detacuerds } \\
\text { aid arairds }\end{array}$ & De axerss & $\begin{array}{l}\text { Congilitanornt: } \\
\text { de averndo }\end{array}$ \\
\hline
\end{tabular}

ais

1. Me prefocto conitantemente ti titor alcansando mis metat

2. Pienso en ranas maceras de resolver un peoblema antes de respondeslo

3. Intento vbikzar citratepas çue me han fancionads to el pasads

4 Jientras estudio oeganizo el tiempo para poder acabar la tarea

5. Sor sonteicete de los pantos fueates $y$ debiles de mi ieteligencis

6 Pienso en lo que reahnemte neceito aprender anites de empezat ana tatea

7. Coasdo tecmans ua examen it sosto me ha ado

8. Je propongo objetiros etpecificos antes de empezar una tasea

9. Vor mals detpacio cauds me tecuentro cos anformacos tatea

10. Tenco clato qué tipo de información es mis importante aprender

11. Caside retrelte as problema me preganto in he tenido en caenta todat I lat ogchorts

12. Sor butao para orgatizas informucida

13 Conscimtermente crntro ma aterición en la informaciben çue et importante

14. Uolino cadi ertrategia coe un propbrito erpecifico

15. Aprendo mejor caando ra conotco alro robre el terma

16. Se que etperan los puoteroces que ro apreeda

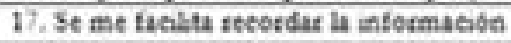

15. Dependiendo de la siruacion utilizo diferentes eatratejias de aperndizaie

19. Caando termino una tarta me preganto ta labia una maneca mat tael de haserts

20. Coando me properaco aptender ua tema. Jo eoratso

21. Reparo peribdicamente para arodame a entender relaciones importunte:

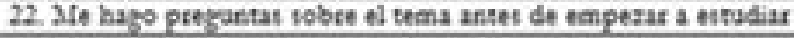

23. Piento en distintas maneras de resolter un problema tescojo la mejor

24 . Cuando termino de eutodiar baro un revamen de lo que he aprendido

25. Fido aroda cuando to entieado alyo

\begin{tabular}{|c|c|c|c|c|}
\hline 1 & 2 & 3 & 4 & 5 \\
\hline 1 & 2 & 3 & 4 & 3 \\
\hline 1 & 2 & 3 & 4 & $\Rightarrow$ \\
\hline 1 & 2 & 3 & 4 & 3 \\
\hline 1 & 2 & 3 & 4 & 5 \\
\hline 1 & 2 & 3 & 4 & 3 \\
\hline 1 & 2 & 3 & 4 & 3 \\
\hline 1 & 2 & 3 & 4 & 3 \\
\hline 1 & 2 & 3 & 4 & 3 \\
\hline 1 & 2 & 3 & + & 3 \\
\hline 1 & 2 & 3 & 4 & S \\
\hline 1 & 2 & 3 & 4 & 5 \\
\hline 1 & 2 & 3 & 4 & 3 \\
\hline 1 & 2 & 3 & 4 & 5 \\
\hline 1 & 2 & 3 & 4 & 5 \\
\hline 1 & 2 & 3 & 4 & 5 \\
\hline 1 & 2 & 3 & 4 & 3 \\
\hline 1 & 2 & 3 & 4 & 5 \\
\hline 1 & 2 & 3 & 4 & 3 \\
\hline 1 & 2 & 3 & 4 & 5 \\
\hline 1 & 2 & 3 & 4 & 5 \\
\hline 1 & 2 & 3 & 4 & 5 \\
\hline 1 & 2 & 3 & 4 & 3 \\
\hline 1 & 2 & 3 & 4 & 5 \\
\hline 1 & 2 & 3 & 4 & 5 \\
\hline
\end{tabular}

Faeultad de Edveación y Ciencias Humasas

Pregrama de Ciencias Naturales

Marstria en Didsetica de las Ciencias Nararales 
26. Pardo motratime para apeender cuando lo necerito 27. Sor convtiente de las estrategias gue utaso cuando entadio

\begin{tabular}{|l|l|l|l|l|}
\hline 1 & 2 & 3 & 4 & 5 \\
\hline 1 & 2 & 3 & 4 & 5 \\
\hline
\end{tabular}

\begin{tabular}{|c|c|c|c|c|c|}
\hline 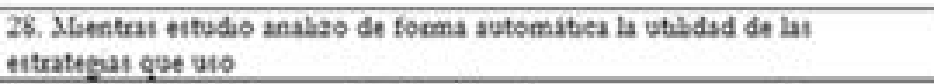 & 1 & 2 & 3 & 4 & 5 \\
\hline $\begin{array}{l}\text { 29. Uto los paston faertes de mi iatelegeneia para eompetanat mis } \\
\text { debildades }\end{array}$ & 1 & $\overline{2}$ & 3 & 4 & 5 \\
\hline $\begin{array}{l}\text { 30. Centro mi atención en el tignificado y la importancia de la infotmación } \\
\text { nueva }\end{array}$ & 1 & $\overline{2}$ & $\overline{3}$ & 4 & 3 \\
\hline $\begin{array}{l}\text { 31. Me invento mis peopion ciemplos para poder entender mejor is } \\
\text { informacion }\end{array}$ & 1 & 2 & 3 & 4 & 5 \\
\hline 32. Me dor cuenta de ti he eatendide algo o no. & 1 & 2 & 3 & 4 & 5 \\
\hline 35. Uthkio de forma automitica eitratecan de aptenchaje utiles & 1 & 2 & 3 & 4 & 3 \\
\hline $\begin{array}{l}\text { 34. Cuando entoy eitadiando, de rez en cuando hapo ana pauta para ver in } \\
\text { eutor entendiendo }\end{array}$ & 1 & 2 & 3 & 4 & 5 \\
\hline 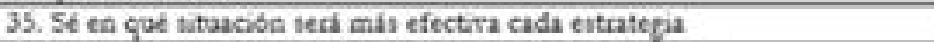 & 1 & 2 & 3 & 4 & 5 \\
\hline $\begin{array}{l}\text { 36. Cuando termino una tares me petzanto kasta qué punto } \\
\text { he consegaldo mis objetivos }\end{array}$ & 1 & 2 & $\overline{3}$ & 4 & 5 \\
\hline 37. MGentras estudio bapo dibojos o diaframas gae me arvden a esteader & 1 & 2 & 3 & 4 & 5 \\
\hline 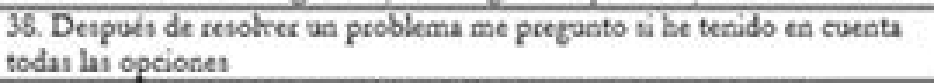 & 1 & 2 & $\overline{3}$ & 4 & 5 \\
\hline 39. Intento expresar con mis propiat palabrat la información naera & 1 & 2 & 3 & 4 & 3 \\
\hline 49. Cuasdo so logto entender ua ptoblema eambis las titratefial & 1 & 2 & 3 & 4 & 5 \\
\hline 41. Utiszo ha estructuar y h organizacion del texto pun comprendet mejoe & 1 & 2 & 3 & 4 & 5 \\
\hline $\begin{array}{l}\text { 42. Leo cuidadosamente bos eruaciados antes de eorpezas } \\
\text { una tases }\end{array}$ & 1 & 2 & $\overline{3}$ & 4 & 5 \\
\hline 43. Mle preganto si lo que estor lerendo estí relacionado con lo que ra sé & 1 & 2 & 3 & 4 & 5 \\
\hline $\begin{array}{l}\text { 44. Cuando stoy coefuedido me pteguete ti ho que toponia eta cottecto o } \\
\text { no }\end{array}$ & 1 & 2 & 3 & 4 & 5 \\
\hline 45. Ogfatazo el tetmpo pasa lopat mejot mis objetros & 1 & 2 & 3 & 4 & 5 \\
\hline 45. Aprendo mis cuando me interena el tema & 1 & 2 & 3 & 4 & 5 \\
\hline 47. Cuasdo estudio inteato hactulo por etapas & 1 & 2 & 3 & 4 & 5 \\
\hline 45. Sle fijo min en el sentido global que en el etpecifico & 1 & 2 & $\sqrt{3}$ & 4 & 5 \\
\hline 49. Cunsdo aprendo algo suero me pregunto ti lo entendo bien o & 1 & 2 & 3 & 4 & 5 \\
\hline $\begin{array}{l}\text { 50. Cuando termino una tated at preganto si he aptendido lo miximo } \\
\text { potible }\end{array}$ & 1 & 2 & $\sqrt{3}$ & 4 & 5 \\
\hline 51. Cuando ha información nueva es confusa, me detengo $r$ la expaso & 1 & 2 & 3 & 4 & 5 \\
\hline 32. Met detenpo r velto cuando eitor confundudo & 1 & 2 & 3 & 4 & 5 \\
\hline
\end{tabular}

\section{Gracias por tus respuestas!}

Por faver escribe to neeble ecenpieto: 